\title{
HOXA5 inhibits the proliferation and neoplasia of cervical cancer cells via downregulating the activity of the Wnt/ $\beta$-catenin pathway and transactivating TP53
}

\author{
Hong-Mei Ma ${ }^{1,2}$, Nan Cuil ${ }^{1,2}$ and Peng-Sheng Zheng ${ }^{1,2}$
}

\begin{abstract}
HOXA5 is considered a regulator involved in embryonic development and cellular differentiation and a tumor suppressor. Nevertheless, its biological role in cervical carcinoma is still unclear. In the present study, immunohistochemistry showed that HOXA5 expression gradually decreased as the degree of cervical lesions deepened. Ectopic expression of HOXA5 restrained cell proliferation, decreased cell viability, and inhibited tumor formation in vitro and in vivo. Furthermore, the expression of HOXA5 could arrest cell cycle from G0/G1 to $S$ phase. RNA-seq revealed that p21 and cyclinD1 were involved in this process. Moreover, the gene set enrichment analysis and the TOP/FOP reporter assay both suggested that HOXA5 could restrain the activity of the Wnt/ $\beta$-catenin pathway. Further study using dual-luciferase reporter assay and quantitative chromatin immunoprecipitation assay demonstrated that HOXA5 could directly bind to the TAAT motif within the promoter of TP53 by its HD domain and transactivate TP53, which can upregulate p21. Altogether, our data suggest that HOXA5 inhibits the proliferation and neoplasia via repression activity of the Wnt/ $\beta$-catenin pathway and transactivating TP53 in cervical cancer.
\end{abstract}

\section{Introduction}

Cervical carcinoma ranked 4th in both morbidity and mortality in all cancer types in females globally ${ }^{1}$. According to the latest cancer statistics, there were over 569,000 new cervical cancer cases and 311,000 deaths in $2018^{1}$. The main cause of cervical cancer is considered to be human papillomavirus (HPV) infection ${ }^{2,3}$, but not every patient with infection of HPV develop cervical cancers, only a small proportion of people develop cervical cancer. This phenomenon indicates that there are other elements participating in cervical carcinogenesis ${ }^{4}$.

\footnotetext{
Correspondence: Peng-Sheng Zheng (zpsheng@mail.xjtu.edu.cn)

${ }^{1}$ Department of Reproductive Medicine, The First Affiliated Hospital of Xi'an Jiaotong University, Xi'an 710061 Shaanxi, People's Republic of China

${ }^{2}$ Section of Cancer Stem Cell Research, Key Laboratory of Environment and Genes Related to Diseases, Ministry of Education of the People's Republic of

China, Xi'an 710061 Shaanxi, People's Republic of China

These authors contributed equally: Hong-Mei Ma, Nan Cui

Edited by I. Amelio
}

Recent studies indicated stem cell-related genes are related with the development of cervical cancer ${ }^{5,6}$. Previous studies in our laboratory demonstrated that dysfunction

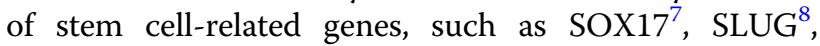
$\mathrm{KLF} 4{ }^{9}, \mathrm{GDF} 15^{10}$, DAX $1^{11}$, and $\mathrm{EZH} 2^{12}$ may participate in cervical carcinogenesis. Therefore, there is a compelling need to explore the underlying mechanism of stem cellrelated genes in cervical cancer.

HOXA5 is a stem cell-related gene and is a member of homeobox gene clusters. HOXA5 is an important development regulator gene that participates in the development of the respiratory system ${ }^{13-15}$. HOXA5 is also considered a marker of terminal differentiation that exerts mutual antagonism with Wnt signaling ${ }^{16}$. Furthermore, HOXA5 was widely studied in esophageal cancer $^{17}$, gastric cancer ${ }^{18}$, renal cancer ${ }^{19}$, melanoma ${ }^{20}$, breast cancer ${ }^{21}$, and colorectal cancer ${ }^{16}$. To the best of our knowledge, the

\section{(c) The Author(s) 2020}

(c) (i) Open Access This article is licensed under a Creative Commons Attribution 4.0 International License, which permits use, sharing, adaptation, distribution and reproduction c. in any medium or format, as long as you give appropriate credit to the original author(s) and the source, provide a link to the Creative Commons license, and indicate if changes were made. The images or other third party material in this article are included in the article's Creative Commons license, unless indicated otherwise in a credit line to the material. If material is not included in the article's Creative Commons license and your intended use is not permitted by statutory regulation or exceeds the permitted use, you will need to obtain permission directly from the copyright holder. To view a copy of this license, visit http://creativecommons.org/licenses/by/4.0/. 
molecular mechanisms of HOXA5 in the development of cervical carcinoma are mostly unclear.

In the present study, we demonstrate that HOXA5 is deregulated in cervical lesions. Ectopic expression of HOXA5 suppresses the proliferation and neoplasia of cervical cancer cells via repressing the activity of the Wnt/ $\beta$-catenin pathway and transactivating TP53.

\section{Materials and methods \\ Clinical samples}

A total of 42 cervical cancer (CC), 28 high-grade squamous intraepithelial lesion (HSIL), and 55 normal cervix (NC) tissues were gleaned from NO.215 Hospital of Shaanxi Nuclear Industry from January 2015 to December 2018. These patients did not receive immunotherapy, chemotherapy, or radiotherapy. The histological classifications and clinical stage were performed on the basis of FIGO.

\section{Immunohistochemistry and immunocytochemistry}

The technical process of immunohistochemistry (IHC) was performed as previously described ${ }^{7}$. The HOXA5 staining strength was categorized into different groups depending on the positive cell percentage and positive cell staining density. The positive cell percentage was categorized into five grades: $0-3 \%(0), 3-25 \%(1)$, $26-50 \%$ (2), $51-75 \%$ (3), and $76-100 \%$ (4). The positive cell staining density was also categorized into four grades: negative (0), weak brown (1), moderate brown (2), and strong brown (3). The final immunohistochemical score was calculated as follows: immunoreactivity score (IRS) = intensity score $\times$ positive score. The final IRS were categorized into three groups: negative $(\leq 3)$, weak positive $(>3$ but $\leq 6)$, and strong positive $(>6)$. The antibodies used were as follows: anti-HOXA5 (1:100, sc-365784, Santa Cruz); anti-Ki67 (1:100, sc-23900, Santa Cruz); anticyclinD1 (1:100, sc-8396, Santa Cruz); anti-p21 (1:50, sc817, Santa Cruz); anti- $\beta$-catenin (1:50, sc-7963, Santa Cruz); anti- p53 (1:50, sc-7963, Santa Cruz). The technical process of immunocytochemistry was performed as previously described ${ }^{22}$.

\section{Western blot}

Fresh tissues and adherent cells were lysed with lysis buffer (the composition of lysis buffer was described previously ${ }^{7}$ ), which included protease inhibitor cocktail (Roche Diagnostics, USA) for $30 \mathrm{~min}$ on ice. After centrifugation, the protein was quantified with BCA quantification (catalogue: 23225, Pierce, USA). Fifty micrograms of cell lysates or tissue lysates were added into sodium dodecyl sulfate-polyacrylamide gel electrophoresis to separate proteins, and then transferred onto the polyvinylidene difluoride (PVDF) membranes. The PVDF membranes were blocked using $5 \%$ non-fat milk for $1 \mathrm{~h}$.
Then the primary antibodies diluted with $5 \%$ non-fat milk were incubated with the membranes overnight at $4{ }^{\circ} \mathrm{C}$. The immunoblot bands were visualized by incubating with horseradish peroxidase (HRP)-conjugated secondary antibodies (Thermo Fisher Scientific). The immunoblot bands were detected with enhanced chemiluminescence reagent (Millipore, Billerica, MA, USA). The primary antibodies used were as follows: anti-HOXA5 (1:1000, sc365784, Santa Cruz); anti-GAPDH (1:1000, sc-47724, Santa Cruz), anti- $\beta$-catenin (1:500, sc-7963, Santa Cruz), anti-c-Myc (1:500, sc-40, Santa Cruz), anti-cyclinD1 (1:1000, sc-8396, Santa Cruz), anti-p53 (1:500, sc-7963, Santa Cruz), and anti-p21(1:1000, \#2947, Cell Signaling Technology). The secondary antibodies used for western blot were HRP-conjugated anti-mouse IgG and antirabbit IgG (Thermo Fisher Scientific).

\section{Cell lines and cell culture}

The human cervical cancer cell lines (HeLa, SiHa, C33A, CaSki, HT-3) were obtained from the American Type Culture Collection (ATCC, Rockville, MD, USA). HeLa, SiHa, and C-33A cells were cultured in highglucose Dulbecco Modified Eagle Medium (SigmaAldrich, St. Louis, MO, USA). RPMI1640 Medium (Sigma-Aldrich, St. Louis, MO, USA) was used to culture CaSki cells. McCoy's 5A Medium (Sigma-Aldrich, St. Louis, MO, USA) was used to culture HT-3 cells. All the cell lines were cultured with the condition in $5 \% \mathrm{CO}_{2}$ at $37^{\circ} \mathrm{C}$.

\section{Cell growth and cell viability assays}

Cells in the logarithmic growth phase were harvested and counted under aseptic conditions. Cells were inoculated into $35 \mathrm{~mm}$ small dishes at $2 \times 10^{4}$ per dish, and cultured with complete medium. When the cells grow to $1,3,5$, and 7 days, cell numbers were counted. The cell growth curve was generated according to the counting result. The cell viability assays were performed using 3(4,5-dimethylthiazole-yl)-2,5-diphenyl tetrazolium bromide (MTT, Sigma-Aldrich). At a wavelength of $490 \mathrm{~mm}$, the absorbance was measured using a microplate reader (Bio-Rad, CA, USA). All the experiments were repeated three times independently.

\section{Tumor xenograft assay}

Four to five-week-old female nude mice (BALB/c) were purchased from SLAC Laboratory Animal Co., Ltd. (Shanghai, China). The mice were divided randomly. The mice were bred in a specific pathogen-free condition in which the temperature is at $22-25^{\circ} \mathrm{C}$ and the humidity is at $40-50 \%$. The cells in the logarithmic growth phase were counted under sterile conditions. The cell suspension $\left(5 \times 10^{5}\right)$ was injected subcutaneously on the back of nude mice, and the tumor volume was measured every 
2-3 days. When the nude mice are sacrificed, the tumor tissue is stripped. The experiment was approved by the Animal Ethics Committee of Xi'an Jiaotong University.

\section{Flow cytometry analysis}

HOXA5-modified cells $\left(4 \times 10^{5}\right)$ were inoculated into $35 \mathrm{~mm}$ dishes. After $24 \mathrm{~h}$, the cells were digested with trypsin and fixed with pre-chilled $75 \%$ ethanol. After washing twice with phosphate-buffered saline (PBS), the cells were resuspended with $200 \mu \mathrm{l}$ PBS, and added with $10 \mu \mathrm{l}$ propidium iodide $(1 \mathrm{mg} / \mathrm{ml}$, Sigma Aldrich, St. Louis, MO, USA) and $20 \mu \mathrm{l}$ RNaseA $(1 \mathrm{mg} / \mathrm{ml}$, Sigma Aldrich, St. Louis, MO, USA), and incubate for $35 \mathrm{~min}$ on ice. FACS Calibur (BD Biosciences, San Jose, CA, USA) was used for flow cytometer, and the data were analyzed by Flow Jo.

\section{RNA isolation and quantitative RT-PCR (qRT-PCR)}

Total RNA was isolated from cells using RNAiso reagent (Takara, Osaka, Japan), and cDNA was reverse transcripted using PrimeScript RT reagent Kit (Takara, Osaka, Japan). The real-time PCR amplification using Total cDNA as templates, and the SYBR Premix ExTaq II (Takara, Osaka, Japan) was used for real-time quantitative PCR. GAPDH was used as an internal control. Real-time quantitative PCR was performed in triplicate for each sample using TianLong TL988 System (TianLong, Xi'an, China). Results were analyzed using the MED-TL-4CH software. The primers used for real-time quantitative PCR are listed in Table S2.

\section{RNA preparation and RNA-seq}

The RNA of three SiHa-GFP and three SiHa-HOXA5 cell lines were isolated using RNAiso (Takara, Osaka, Japan), and all RNA samples meet a criterion to quality control. The RNA-seq was conducted using the BGISEQ500 platform. The average output data of each sample was $23.98 \mathrm{Mb}$. The clean reads of Q30 of the RNA samples were all over $90 \%$. The average contrast ratio of sample to genome was $94.02 \%$, and the contrast ratio of samples to gene set was $83.37 \%$. 17,901 genes were detected. The data was analyzed using NOISeq method with the threshold $\log 2$ fold-change $>2$.

\section{Plasmids and cell transfection}

The CDS of HOXA5 was cloned by PCR and were inserted into pIRES2-AcGFP (Clontech, Mountain View, CA) to construct pIRES2-AcGFP-HOXA5 expressing plasmids. HOXA5-specific shRNAs were cloned and inserted into pGPU6/GFP/Neo (GenePharma, Shanghai, China) to restrain the expression of HOXA5.

The pSpCas9(BB)-2A-GFP (PX458) plasmid (Plasmid \#68370) containing SpCas9 and pSpCas9(BB)-2A-Puro (PX459) plasmid (Plasmid \#48139) were purchased from Addgene (Cambridge, MA, USA). The single guide RNA targeting the first exon of HOXA5 were designed using the website (http://crispr.mit.edu/).

To obtain the stable transfection cell lines, the plasmids were transfected into cervical cancer cell lines using Lipofectamine 2000 reagent (Invitrogen, Carlsbad, CA, USA) according to the manufacturer's instructions. G418 (MCE, New Jersey, CA, USA) was added into the media of transfected cells for selection with stress.

\section{Luciferase reporter assay}

The fragments of TP53 promoter were amplified and cloned into pGL3.0-Basic Vector (Promega, Madison, WI, USA) for the luciferase reporter plasmids. The fragments were verified using Sanger sequencing. HOXA5-modified cells were seed into 24-well-plate $\left(8 \times 10^{5}\right)$ and cotransfected with above plasmids and pRL-TK plasmids (Promega) using Lipofectamine 2000 (Invitrogen). Cells were collected $48 \mathrm{~h}$ post-transfection, and luciferase activities were detected by luminometer (Promega) using the Dual-Luciferase Assay Kit (Promega, Madison, WI). Data were analyzed as relative luciferase activity (Firefly luciferase activity/Renilla luciferase activity). Each experiment was performed in triplicate.

\section{Quantitative chromatin immunoprecipitation}

The ChIP assay was used to evaluate transcription factor HOXA5 binding sites using the EZ-Magna ChIP Assay Kit (Millipore, Darmstadt, Germany). The protocol was performed as described previously ${ }^{8}$. The chromatin-protein complexes were immunoprecipitated with $10 \mu \mathrm{g}$ of antiHOXA5 antibody (sc-365784, Santa Cruz, CA, USA). The DNA fragments obtained from that were then used as templates for real-time PCR. Each sample was performed in triplicate, and the fold enrichment ratio was measured as the value of the ChIP sample vs. the corresponding input sample.

\section{Statistical analysis}

Statistical analysis was conducted using SPSS 19.0 software (SPSS Inc., Chicago, IL). All data are shown as means \pm standard deviation of the mean (SD). Gene expression in tumor tissues and their controls were compared by the unpaired $t$-test, and paired samples were compared by the paired $t$-test. In all tests, $p<0.05$ is regarded as statistically significant.

\section{Results}

HOXA5 is frequently downregulated in cervical cancers

To understand the expression pattern of HOXA5 in cervical cancer, HOXA5 protein expression was detected in NC $(n=42)$, HSIL, $(n=28)$, and CC $(n=55)$ by IHC. The results revealed that HOXA5 localized in the nucleus, and representative images are shown in Fig. 1a. The total HOXA5-positive (strongly positive and weakly positive) rate decreased from $76.2 \%$ in $\mathrm{NC}$ samples to $28.6 \%$ in 


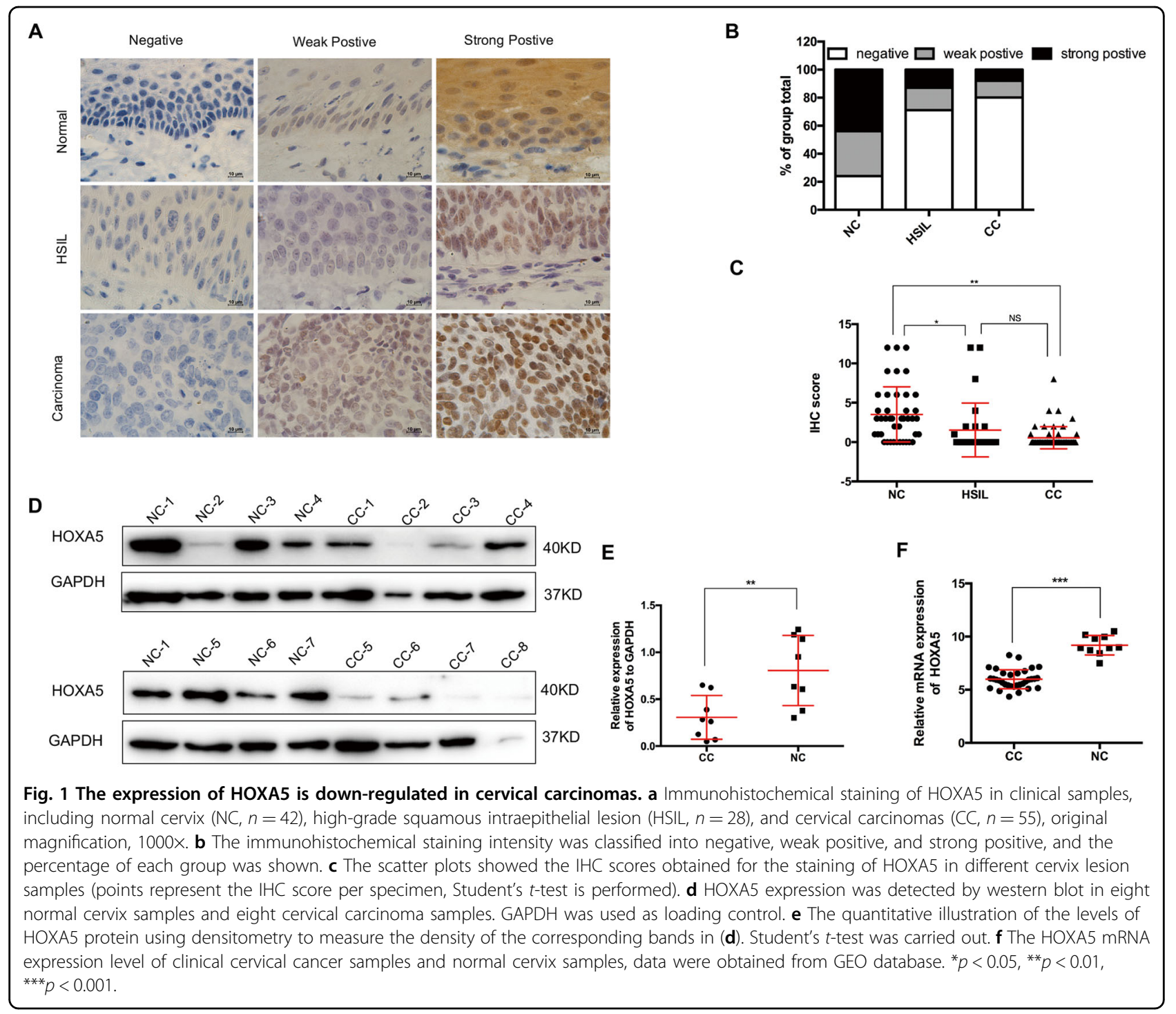

HSIL and then $20.0 \%$ in CC samples (Table S1 and Fig. 1b, $p<0.05)$. Significant differences were observed between either of the two groups (Table S1, NC vs. HSIL, $p<0.05$; $\mathrm{NC}$ vs. CC, $p<0.001$; HSIL vs. CC, $p<0.001$ ). Additionally, the analysis of IRS showed that HOXA5 staining was $3.52 \pm 0.54$ in NC, $1.54 \pm 0.65$ in HSIL, and $0.56 \pm 0.19$ in CC (Fig. 1c). Moreover, western blot analysis was performed in eight NC tissue samples and eight CC tissue samples (Fig. 1d). The average HOXA5 expression level in NC $(0.8051 \pm 0.1321, n=8)$ was two times higher than that in CC $(0.3062 \pm 0.08264, n=8)$ (Fig. 1e). Furthermore, data from the GEO database (GDS2416, GDS3233, and GDS3292) also showed that HOXA5 expression in CC samples was significantly lower than that in NC samples (Fig. 1f). These findings suggest that HOXA5 may play a suppressive role in the carcinogenesis and development of cervical cancer.

\section{HOXA5 inhibits the proliferation of cervical cancer cells} in vitro

The expression of HOXA5 protein in cervical cancer cell lines was evaluated by western blot and immunochemistry. As shown in Fig. 2a, b, HOXA5 was highly expressed in C$33 \mathrm{~A}$ cells, and was expressed to a lower degree in HeLa, $\mathrm{SiHa}$, and HT-3 cells, and almost no expression was detected in CaSki cells. Therefore, HOXA5 was stably overexpressed in HeLa (Fig. 2c) and SiHa cells (Fig. 2f). In addition, HOXA5 was knocked down using small hairpin RNA and knocked out in C-33A cells using CRISPR-Cas9mediated gene editing (Figs. 2i, 1 and S1A, B). The overexpression, knockdown, and knockout efficacies of HOXA5 were confirmed by western blot (Fig. 2c, f, i, l).

As shown in Fig. 2d, g, HeLa and $\mathrm{SiHa}$ cells overexpressing HOXA5 (HeLa-HOXA5, SiHa-HOXA5) exhibited markedly lower proliferation ability than their 


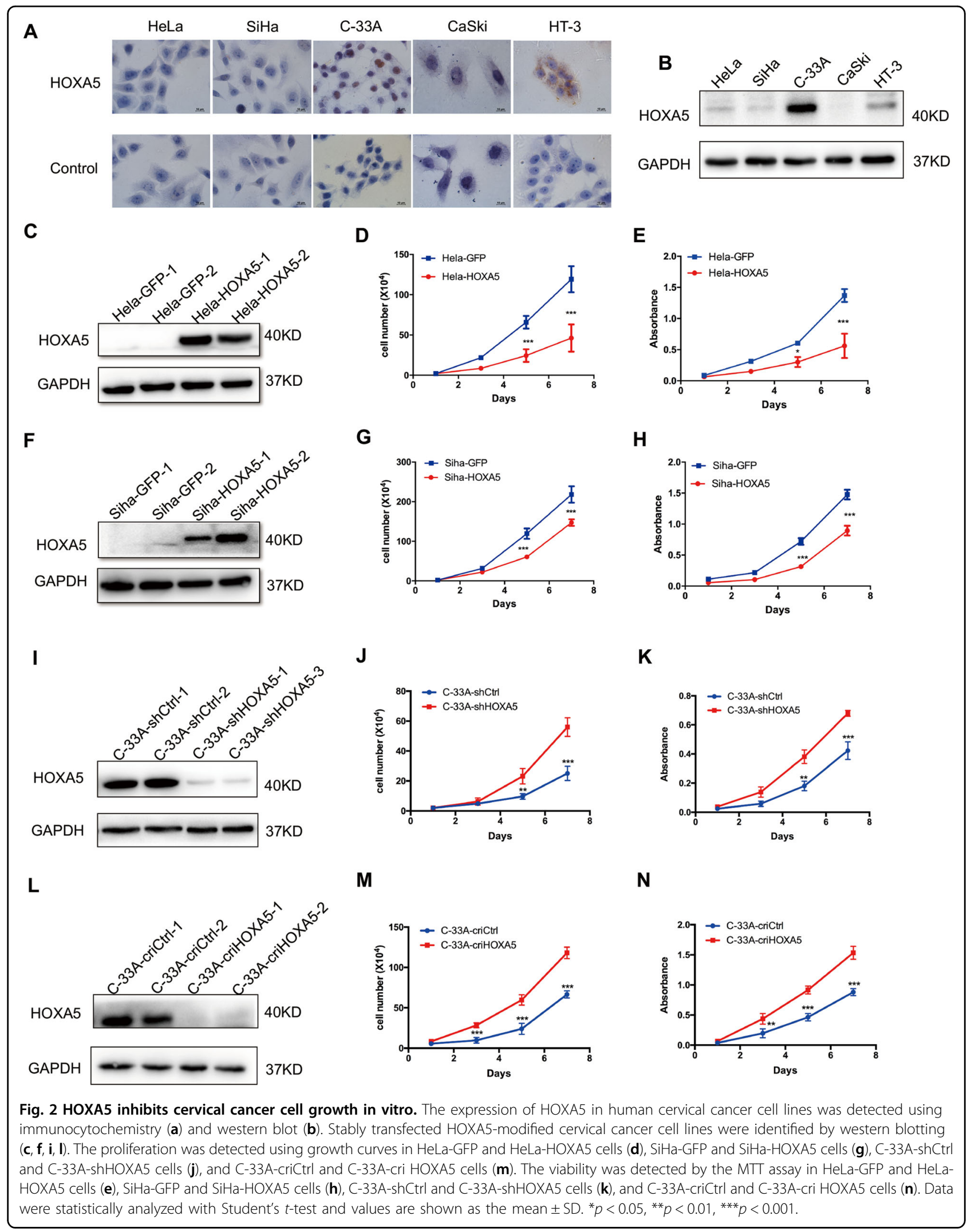


control cells (HeLa-GFP, SiHa-GFP, $p<0.001$ ). In addition, the viability of HeLa-HOXA5 and SiHa-HOXA5 cells was also significantly lower than that of their control cells (HeLa-GFP, SiHa-GFP, $p<0.001$ ). Conversely, knockdown and knockout of HOXA5 in C-33A cells resulted in a remarkable increase in cell proliferation and viability (Fig. 2 j, $k, m, n, p<0.001$ ). Altogether, these findings demonstrate that HOXA5 inhibits the proliferation of cervical cancer cells in vitro.

\section{HOXA5 inhibits tumor formation and the proliferation of} cervical cancer cells in vivo

To explore the function of HOXA5 in vivo, xenograft assays were performed in nude mice, and the development of solid tumors was monitored. A total of $5 \times 10^{5}$ HOXA5-modified cells (HeLa-HOXA5, SiHa-HOXA5) and their control cells (HeLa-GFP, SiHa-GFP) were injected subcutaneously into the nude mice (Fig. 3a, e). It was obvious that the tumors developed from HeLaHOXA5 cells grew slower (Fig. 3b, $p<0.001$ ) and were lighter (Fig. 3c, $0.9876 \pm 0.1811$ vs. $0.04345 \pm 0.01577, p<$ 0.001; Fig. S2A) than tumors developing from HeLa-GFP cells. Moreover, the survival of free tumors in HOXA5overexpressing cells was significantly longer than that of their control cells (Fig. 3d, $p<0.001$ ). Similar results were obtained in SiHa-HOXA5 cells (Fig. 3f, $p<0.001$; Fig. 3g, $0.5842 \pm 0.06465$ vs. $0.05253 \pm 0.02261, p<0.001$; Fig. 3 h, $p<0.01$; Fig. S2B). These findings demonstrated that HOXA5 could inhibit neoplasia and tumor growth of cervical cancer cells in vivo.

To test whether the tumor suppression function of the HOXA5 protein was due to its ability to suppress cell proliferation, the expression of Ki67, which is an important cell proliferation marker, was evaluated by immunohistochemistry in mice xenograft tissues. Tumor tissue formed by HOXA5-overexpressing cells showed much more HOXA5 staining (Fig. 3i, j, $2.83 \pm 0.75$ vs. $9.23 \pm 1.55$, $p<0.001 ; 1.53 \pm 0.67$ vs. $8.51 \pm 1.43, p<0.001$ ) but weaker Ki67 staining (Fig. 3i, j, $10.50 \pm 0.67$ vs. $2.13 \pm 1.10, p<$ $0.001 ; 11.00 \pm 0.63$ vs. $0.83 \pm 0.48, p<0.001$ ) than those formed by the control cells. These findings suggest that HOXA5 could suppress neoplasia and the development of cervical cancer, and the mechanism of this suppression may be due to its ability to inhibit cell proliferation.

\section{HOXA5 inhibits the proliferation of cervical cancer cells by arresting the cell cycle process from $\mathrm{G0} / \mathrm{G} 1$ to $\mathrm{S}$ phase}

To understand the mechanism of how HOXA5 protein inhibited the proliferation of cervical cancer cells, fluorescence-activated cell sorting was performed to analyze the cell cycle of HOXA5-modified cells and their control cells. As shown in Fig. 4a, b, ectopic expression of HOXA5 led to a significant increase in the percentage of cells in G0/G1 phase $(62.28 \pm 2.57 \%$ vs. $71.02 \pm 7.00 \%$, $p<0.05)$ and a decrease in the percentage of cells in S/G2/ $M$ phase $(36.64 \pm 2.27 \%$ vs. $23.94 \pm 71.96 \%, p<0.001)$. A similar result was obtained in $\mathrm{SiHa}$ cells (Fig. 4c, d, $56.22 \pm 3.61 \%$ vs. $63.60 \pm 1.94 \%, p<0.001 ; 45.22 \pm 3.45 \%$ vs. $37.8 \pm 1.84 \%, p<0.001)$. Conversely, knockdown and knockout of HOXA5 in C-33A cells dramatically reduced the percentage of cells in G0/G1 phase (Fig. 4e-h, $40.58 \pm$ $7.76 \%$ vs. $33.06 \pm 6.34 \%, \quad p<0.05 ; \quad 47.70 \pm 1.19 \%$ vs. $38.97 \pm 4.29 \%, p<0.05$ ), but increased the percentage of S/G2/M phase cells (Fig. $4 \mathrm{e}-\mathrm{h}, 60.98 \pm 7.63 \%$ vs. $65.93 \pm$ $7.70 \%$, NS; $52.33 \pm 1.85 \%$ vs. $61.07 \pm 4.31 \%, p<0.05$ ). These results suggest that HOXA5 inhibits the proliferation of cervical cancer cells by arresting the cell cycle process from G0/G1 to $\mathrm{S}$ phase.

\section{HOXA5 arrests the cell cycle transition from G0/G1 to $\mathrm{S}$ phase through cyclinD1 and p21}

The cell cycle is regulated by a set of cell cycle-related molecules. To understand HOXA5-induced phenotype changes, we performed RNA-seq (BGI500) in three SiHaHOXA5 cell lines and three SiHa-GFP cell lines. The results of RNA-seq showed that two cell cycle-related molecules, p21 (the coding gene is called CDKN1A) and cyclinD1 (the coding gene is called CCND1), changed significantly among the 395 most differentially expressed genes (Fig. 5a). As shown in Fig. 5b, c, the mRNA levels of CCND1 were decreased in HOXA5-overexpressing cells and increased in HOXA5-knockdown and HOXA5knockout cells. Conversely, the mRNA levels of CDKN1A were increased in HOXA5-overexpressing cells and decreased in HOXA5-knockdown and HOXA5knockout cells (Fig. 5b, c). These data suggested that HOXA5 suppressed the expression of CCND1 and promoted the expression of CDKN1A at the transcriptional level. Consistent with the mRNA results, the $\mathrm{p} 21$ protein was significantly increased in HOXA5-overexpressing cells and xenografts derived from HOXA5overexpressing cells. Conversely, cyclinD1 protein expression was decreased in HeLa-HOXA5 and SiHaHOXA5 cells and xenografts derived from these cell lines (Fig. $5 \mathrm{~d}-\mathrm{f}$ ). The results were also supported by IHC assays (Figs. 5g, h and S3A, B). These data suggested that HOXA5 regulated the expression of cyclinD1 and p21 at the translational level. All the above data demonstrate that HOXA5 possibly arrest the cell cycle process from G0/G1 to $\mathrm{S}$ phase through cyclinD1 and $\mathrm{p} 21$.

\section{HOXA5 suppresses the expression of cyclinD1 by inhibiting the activity of the Wnt/B-catenin pathway in cervical cancer cells}

Ordonez-Moran et al. reported that there is a mutual antagonistic relationship between HOXA5 and the Wnt pathway $^{16}$. Since a dual-luciferase reporter assay showed that HOXA5 did not directly bind to the promoter of 


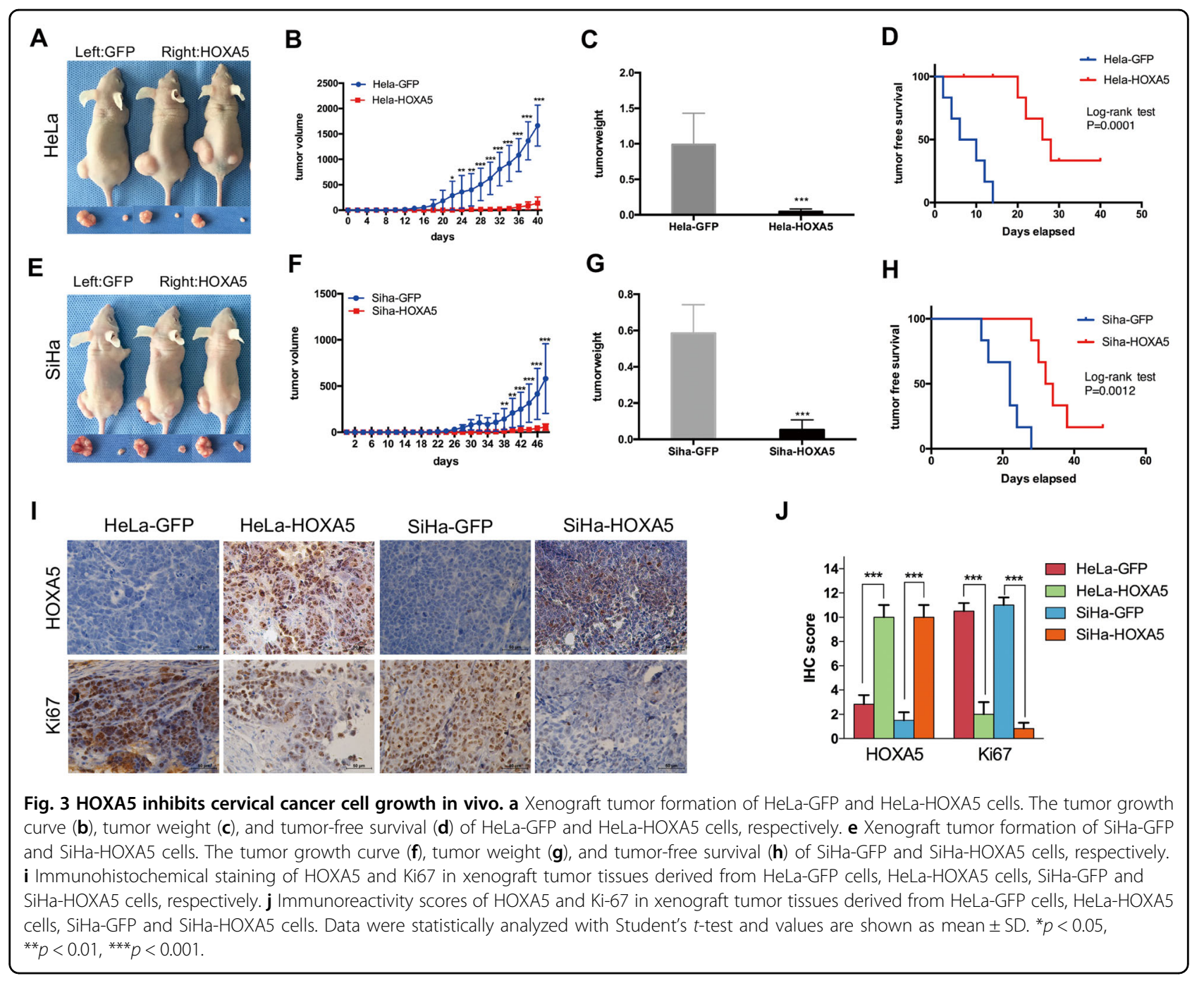

CCND1 (Fig. S4A), we hypothesized that the overexpression of HOXA5 could affect the expression of cyclinD1 through the Wnt pathway. Among the changed genes in RNA-seq, we identified 46 genes which are related with $\mathrm{Wnt} / \beta$-catenin signaling pathway that were differentially expressed (Fig. 6a). A gene set enrichment analysis (GSEA) also indicated that $\mathrm{Wnt} / \beta$-catenin pathway was repressed in SiHa-HOXA5 cells (Fig. 6b). To further detect the changes of $\mathrm{Wnt} / \beta$-catenin pathway, the TOP/FOP flash luciferase reporter assays were conducted. Compared with the control cells, ectopic expression of HOXA5 led to a decrease of TOP flash luciferase reporter activity in HeLa and SiHa cells (Fig. 6c, d). However, knockdown and knockout of HOXA5 increased the activity of the TOP flash luciferase reporter in C-33A cells (Fig. 6e, f). Further study demonstrated that the overexpression of HOXA5 repressed the activity of the TOP flash luciferase reporter in a dose-dependent manner (Fig. S4B). These data demonstrated that the activity of Wnt/ $\beta$-catenin pathway was inhibited by HOXA5 in cervical cancer cell lines. Since the $\mathrm{Wnt} / \beta$-catenin pathway involves a set of molecules, we detected the mRNA and protein levels of the key molecules of the Wnt/ $\beta$-catenin signaling pathway CTNNB1, MYC, CCND1, and GSK3 $\beta$. As Fig. $6 \mathrm{~g}-\mathrm{k}$ shows, the mRNA and protein levels of MYC and CCND1 decreased strongly in HeLa-HOXA5 and SiHa-HOXA5 cells and the xenografts derived from HOXA5-overexpressing cells (Fig. S4C-H). However, the mRNA and protein levels of GSK3 $\beta$ and CTNNB1 did not show any changes after HOXA5 modified. As reported previously, the nuclear accumulation of $\beta$-catenin triggered a downstream molecules cascade. To detect the underlying mechanism, we performed a nuclear separation assay on HOXA5-modified cells. Although total $\beta$-catenin did not show any changes, the distribution of $\beta$-catenin in the nucleus was significantly decreased in HOXA5-overexpressing HeLa and $\mathrm{SiHa}$ cells and was significantly increased in HOXA5-knockdown and 

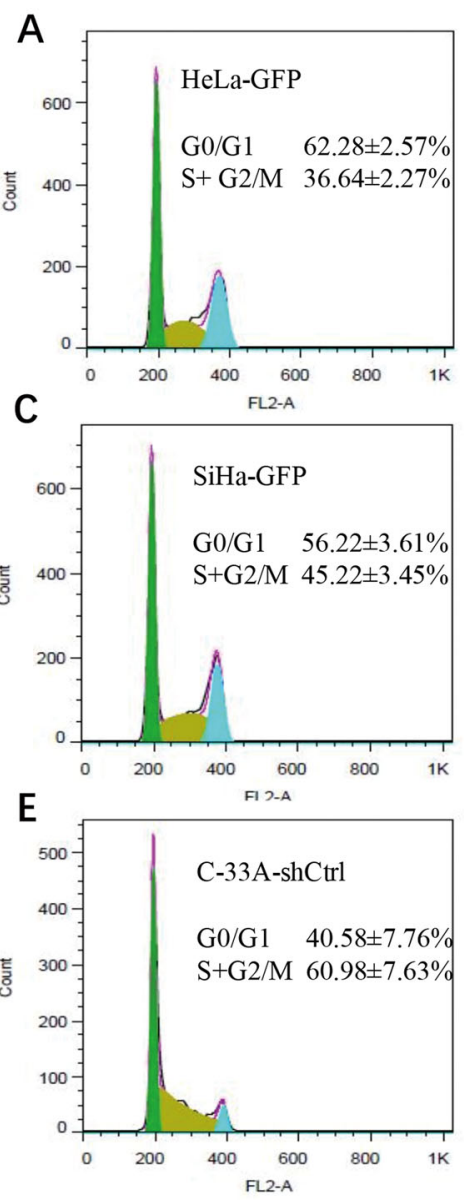

G

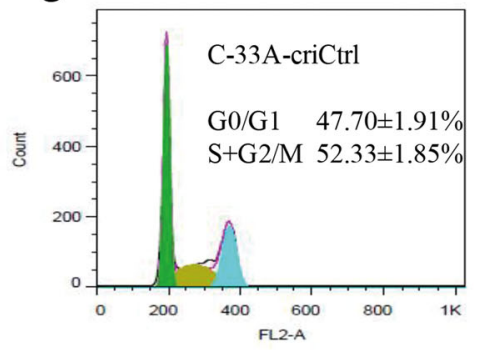

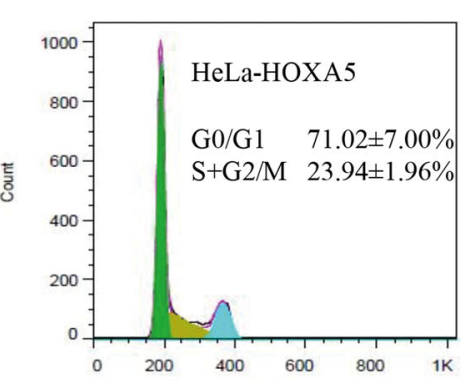
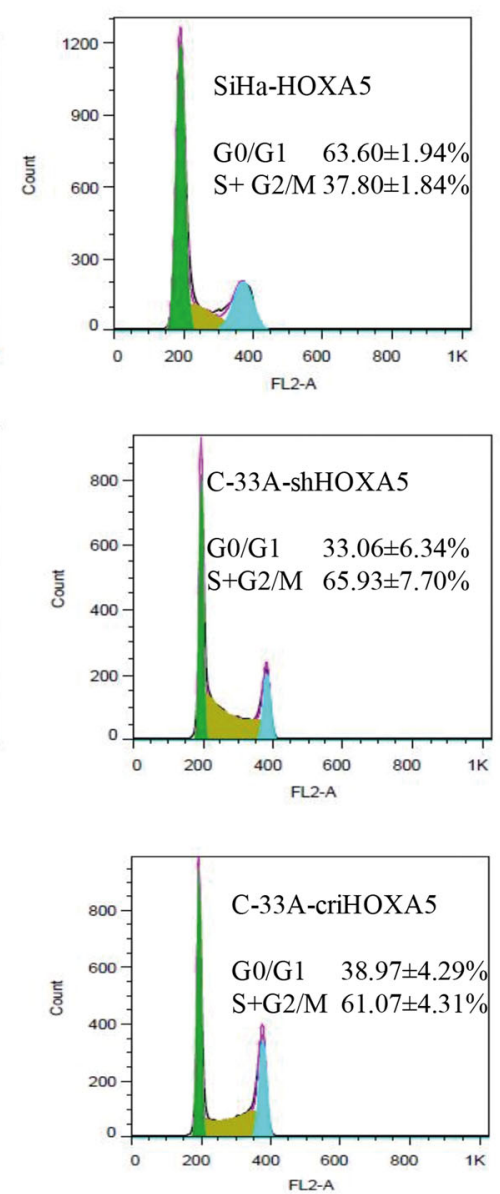

B
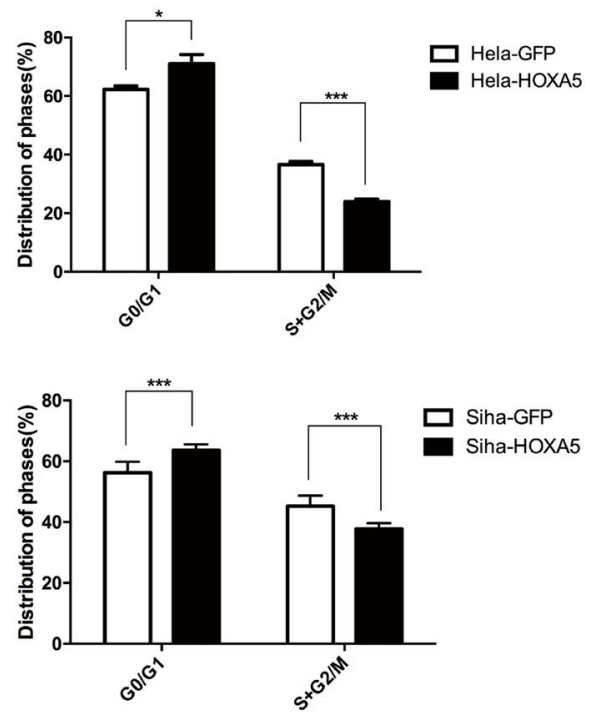

$\mathbf{F}$

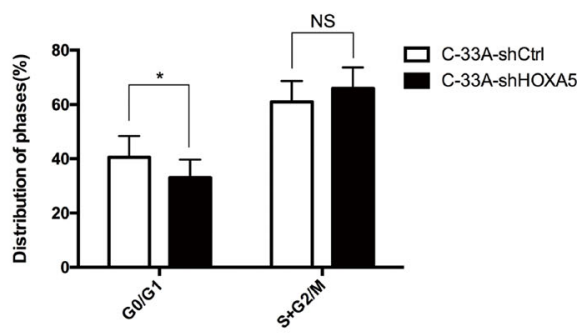

$\mathrm{H}$

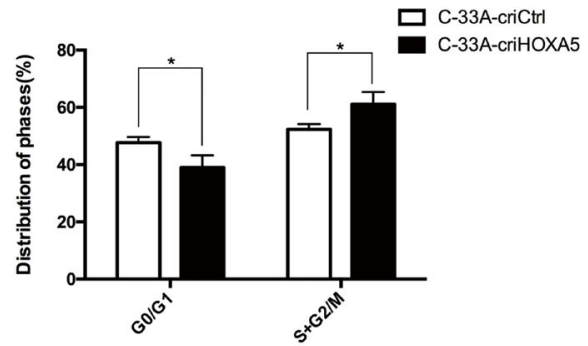

Fig. 4 Expression of HOXA5 in cervical cancer cells impeded cell cycle transition from G0/G1 to S phase. a In the flow cytometry figures, the $y$ axis shows the count of effective cells and the $x$-axis shows the DNA content. Each colored area represents the cells of different phases of cell cycle: green area refers to the cells in the G0/G1 phase, yellow area refers to the cells in the S phase, and blue area refers to the cells in the G2/M phase. The cell cycles of HeLa-GFP and HeLa-HOXA5 cells were analyzed using flow cytometry (a), and a quantitative analysis of the cell cycle are shown (b). The cell cycles of SiHa-GFP and SiHa-HOXA5 cells (c) and the quantitative analysis (d) are shown. The cell cycles of C-33A-shCtrl and C-33A-shHOXA5 cells (e) and the quantitative analysis (f) are shown. The cell cycles of C-33A-criCtrl and C-33A-cri HOXA5 cells ( $\mathbf{g})$ and the quantitative analysis (h) are shown. The data were shown as the mean \pm SD of three independent experiments. Data were statistically analyzed with Student's $t$-test and values are shown as mean $\pm \mathrm{SD}$. ${ }^{*} p<0.05,{ }^{* *} p<0.01,{ }^{* * *} p<0.001$.

HOXA5-knockout C-33A cells (Fig. 6l). Immunochemistry also showed the same results (Fig. $6 \mathrm{~m}$ ). All these data indicate that HOXA5 suppressed the expression of cyclinD1 by inhibiting the activity of the $\mathrm{Wnt} / \beta$-catenin signaling pathway through inhibition of the nuclear translocation of the $\beta$-catenin protein in cervical cancer.
HOXA5 promotes the expression of $\mathrm{p} 21$ by transactivating TP53 through direct binding to its promoter in cervical cancer cells

Since HOXA5 did not bind to the promoter of CDKN1A in HeLa and SiHa cells (Fig. S5A), and p21 is a well-defined downstream factor of $\mathrm{p} 53^{23,24}$, we speculated 


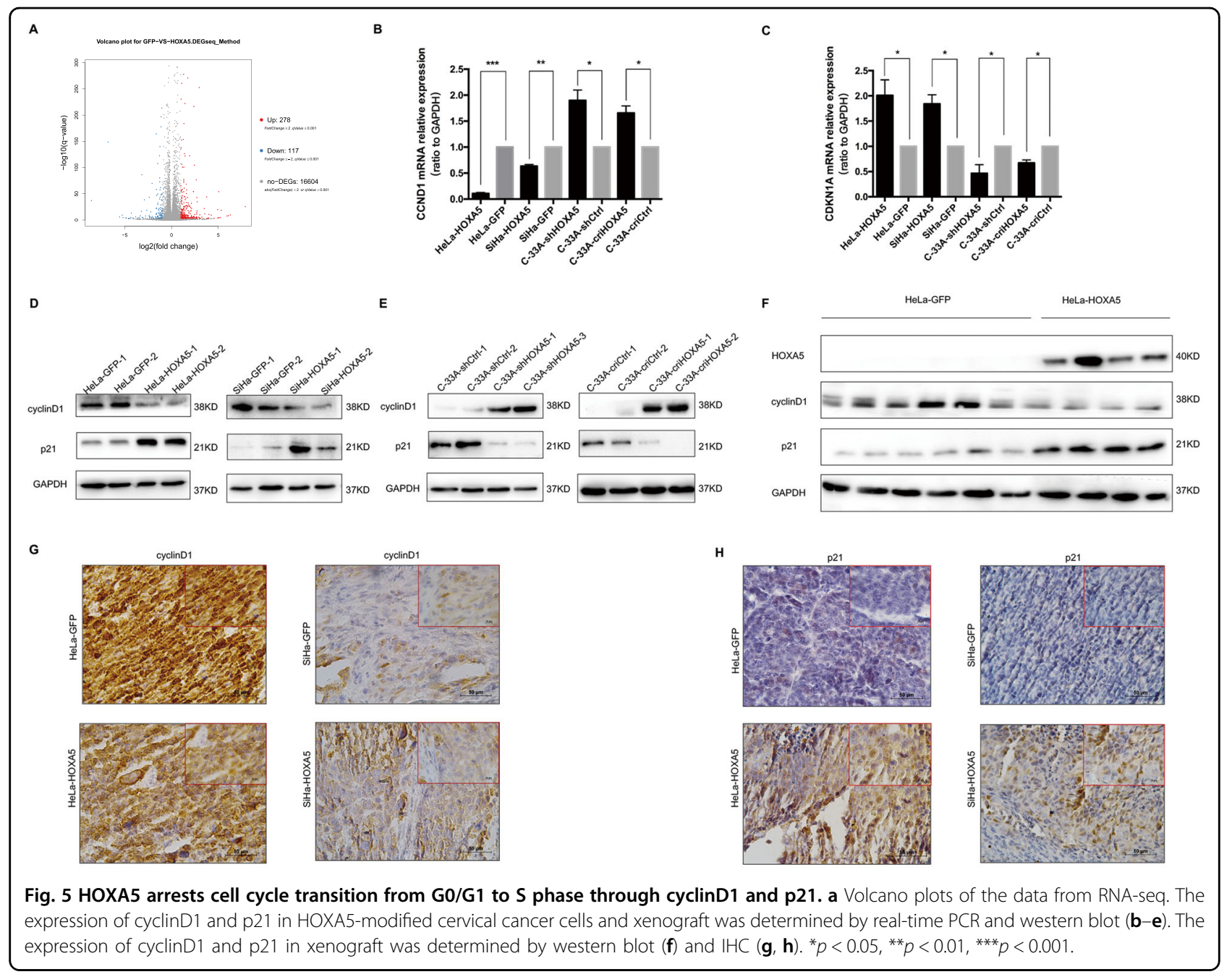

that HOXA5 regulated the expression of p 21 protein by transactivating TP53. The results showed that p53 was up-regulated in HOXA5-overexpressing cells, while it was down-regulated in HOXA5-knockdown and HOXA5knockout C-33A cells, at both transcriptional and translational levels (Figs. 7a-c and S5B). To validate the correlation between HOXA5 and p53, the protein levels of HOXA5 and p53 in clinical cervical cancer specimens were detected by western blot (Fig. $7 \mathrm{~d}$ ). The results suggested that p53 and p 21 were both positively correlated with HOXA5 (Fig. 7e, $r=0.7832, p=0.0002$; Fig. 7f, $r=$ $0.8823, p<0.0001)$. Then, the correlation between HOXA5 and p53 in the GEO database (GDS2416, GDS3233, and GDS3292) was analyzed in a large cohort of cervical cancers. As expected, there was a significant positive correlation between the expression of HOXA5 and the expression of p53 (Fig. $7 \mathrm{~g}, r=0.4118, p<0.0001$ ). To further characterize the relationship between HOXA5 and p53, the HOXA5 plasmid was transfected into HeLa parental cells, and the cells were harvested at different times. The results showed that p53 protein gradually increased as time lasted (Fig. S5C, D).

By analyzing the promoter of TP53 on an online database (http://jaspar.genereg.net), the classical TAAT motif (classical homeobox gene binding motif) was found in the promoter of TP53 (Fig. 7k). Therefore, a dual-luciferase reporter assay was used to determine whether HOXA5 directly binds to the promoter of TP53. We cloned the full-length TP53 promoter $(-1835 /+997 \mathrm{bp})$ into a pGL3.0-basic luciferase plasmid (pGL3.0-TP53). Transient expression of HOXA 5 by the transfection of the luciferase construct into $293 \mathrm{~T}$ cells resulted in a significant induction of luciferase activity driven by the TP53 promoter compared with the control plasmid (Fig. S5E). To explore the core binding region within the TP53 promoter, the promoter of TP53 was divided into eight different lengths, each of which contained HOXA5 binding motifs (P1: $-1835 /+997$; P2: $-1071 /+997$; P3: -557/+997; P4: -380/+997; P5: -277/+997; P6: -55/ +997; P7: +171/+997; and P8: +518/+997). The pGL3.0- 


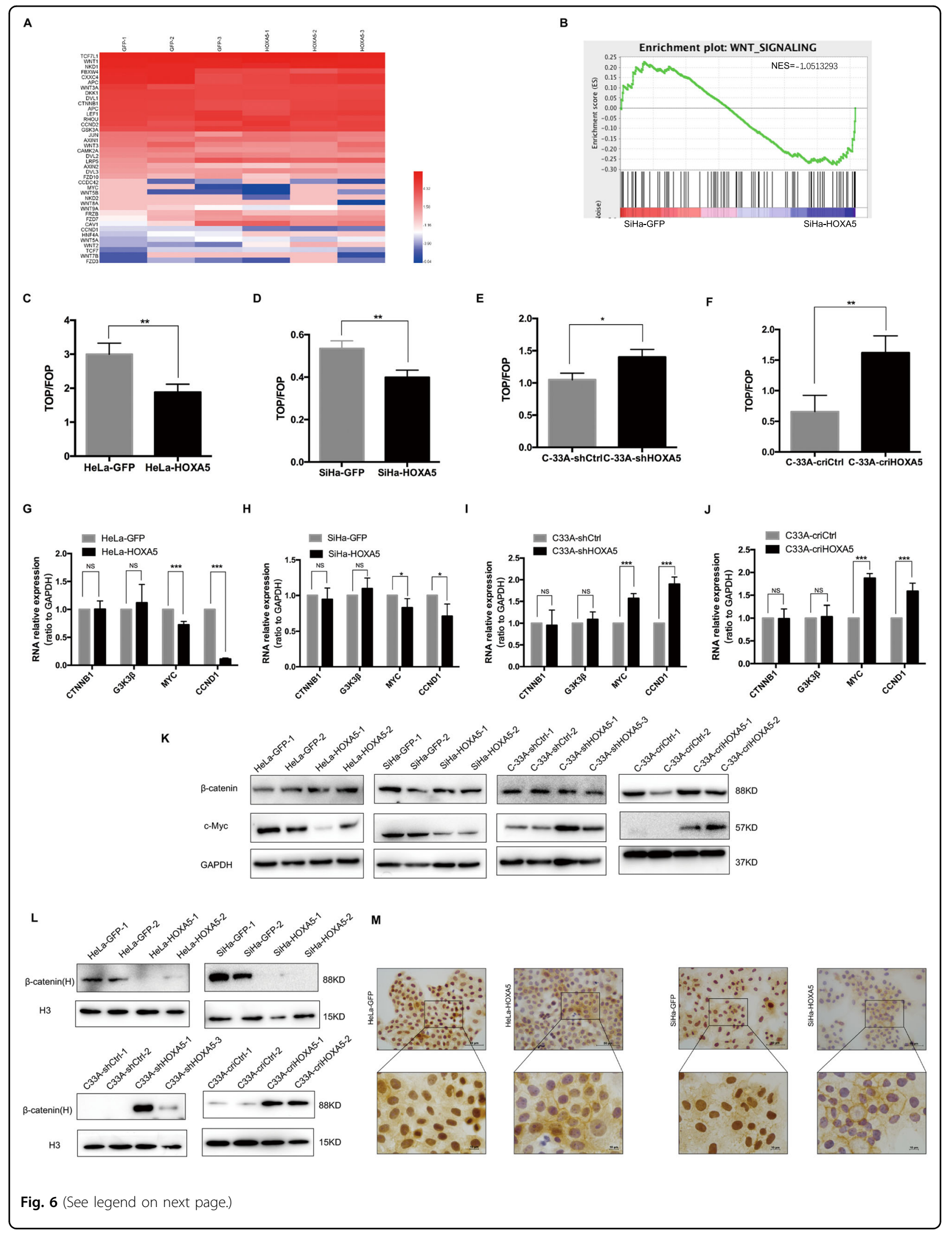


(see figure on previous page)

Fig. 6 HOXA5 inhibited the expression of cyclinD1 via suppressing the Wnt/ $\beta$-catenin pathway in cervical cancer cells. a Heatmap of known genes for Wnt/ $\beta$-catenin pathway in SiHa-GFP cells (left) and SiHa-HOXA5 cells (right) using data from RNA-seq. Data were log 2 normalized. b A gene set enrichment (GSEA) assay using data from RNA-seq. c-f TOP/FOP-Flash reporter assays were carried out in HOXA5-modified cervical cancer cells. $\mathbf{g}$-j Real-time PCR analysis was shown for the mRNA levels of Wnt/ $\beta$-catenin pathway key genes in HOXA5-modified cervical cancer cells. $\mathbf{k}$ The expression of Wnt/ $\beta$-catenin pathway key proteins in HOXA5-modified cervical cancer cells was determined by western blot. The nuclear $\beta$-catenin of HOXA5-modified cervical cancer cell was determined by western blot (I) and immunocytochemistry $(\mathbf{m})$. Data represent mean \pm SD of triplicate experiments and statistical analysis was done with Student's $t$-test. ${ }^{*} p<0.05,{ }^{* *} p<0.01,{ }^{* *} p<0.001$.

basic luciferase plasmid containing different lengths of the TP53 promoter was then transfected into HOXA5modified cells. The results showed that pGL3.0-TP53-P1 and pGL3.0-TP53-P2 exhibited a significant increase in luciferase activity in HeLa-HOXA5 and SiHa-HOXA5 cells compared with their control cells (Fig. 7h, i). These results suggested that the sequence between nucleotides -1071 and -557 in the promoter of TP53 may contain HOXA5 binding sites.

To further determine the direct regulation of TP53 by HOXA5 through binding to its promoter, a ChIP assay was performed in HOXA5-modified cells. RT-PCR applying specific primers to $S 1(-1071$ to -712$)$ and S2 $(-711$ to -581$)$ was conducted to amplify the binding region. The results suggested that HOXA5 strongly bound to S2 in the TP53 promoter region in both HeLa and $\mathrm{SiHa}$ HOXA5-overexpressing cells (Fig. 7j). In accordance with the analysis in the JASPAR CORE database, $5^{\prime}$-TAAT $-3^{\prime}$ was located between nucleotides -693 and -675 in $\mathrm{S} 2$ of the P2 promoter (Fig. $7 \mathrm{k}$ ).

Among the HOXA5 binding motifs, $5^{\prime}-\mathrm{TAAT}-3^{\prime}$ is the most important. To further evaluate the importance of this motif, a mutant pGL3.0-TP53 plasmid with mutated $5^{\prime}$-TAAT-3' to $5^{\prime}$-TAAG-3' was constructed (pGL3.0TP53-P2-mut). A decrease in TP53 promoter activity was shown after the transient expression of HOXA5 by the transfection of the mutant luciferase construct in 293T cells (Fig. S5F). It has been reported that the HOX family expresses a conserved HD domain that functions in binding $\mathrm{DNA}^{25}$. Therefore, an HD domain-deleted mutant of the HOXA5 plasmid (HOXA5- $\triangle H D$ ) was constructed. The co-transfection of the HOXA5- $\triangle H D$ plasmid with the pGL3.0-TP53 plasmid failed to show an increase in luciferase activity (Fig. S5G). The study by Teo and colleagues also obtained the same result in breast cancer cell lines ${ }^{21}$. These data demonstrated that HOXA5 promoted the expression of the p21 protein by directly binding to the $5^{\prime}$-TAAT- $3^{\prime}$ motif in the promoter of TP53 through its HD domain in cervical cancer cells.

\section{Upregulating $\beta$-catenin or inhibiting p53 only partly rescues the proliferation inhibitory effect of HOXA5}

The results above suggested that the suppressed function of HOXA 5 was mediated by cyclinD1 and p21, but it is unclear whether there is crosstalk between the Wnt pathway and p53. Thus, we tested the effect of activating the Wnt/ $\beta$-catenin pathway in HOXA5-modified cervical cancer cells via transient transfection of the $\beta$-catenin plasmid. The results showed that the proliferationinhibiting ability of HOXA5 in cervical cancer cells was partly abolished upon overexpression of $\beta$-catenin in both HOXA5-overexpressing HeLa and SiHa cells (Fig. S6A, B). Moreover, we also found that the overexpression of $\beta$-catenin reversed the inhibitory effect of HOXA5 on cMyc and cyclinD1 proteins but did not affect the protein levels of p21 and p53 (Fig. S6C). Furthermore, the specific p53 inhibitor Pifithrin- $\alpha$ (PFT $\alpha$ ) inhibited the expression of p21, as well as p53 protein, and partly rescued the proliferative ability of HOXA5-overexpressing HeLa and SiHa cells. (Fig. S6D, E). However, the proteins in the $\mathrm{Wnt} / \beta$-catenin pathway were not affected by p53 inhibition (Fig. S6F). All these data suggested that there is possibly no crosstalk between the Wnt pathway and p53.

\section{Correlation analysis of the expression of HOXA5 with the Wnt pathway and p53 in human cervical cancer tissues}

To further confirm the relationship between HOXA5 and the proteins in $\mathrm{Wnt} / \beta$-catenin pathway and p53 in cervical cancer specimens, the expression of HOXA5, $\beta$-catenin, cyclinD1, p53, and p21 was detected by IHC in 15 cervical cancer specimens (Fig. 8a). As expected, HOXA5 expression was negatively correlated to the expression of $\beta$-catenin and cyclinD1 (Fig. 8b, $r=$ $-0.5931, p<0.05$; Fig. 8c, $r=-0.5428, p<0.05$ ), while HOXA5 expression was positively correlated with the expression of p53 and p21 (Fig. 8d, $r=0.5309, p<0.05$; Fig. 8e, $r=0.6204, p<0.05)$. These results implied that HOXA5 possibly affected the proliferation of cervical cancer cells by both the Wnt/ $\beta$-catenin/cyclinD1 and p53/ p21 pathways.

\section{Discussion}

HOXA5 has been reported to be involved in embryo development ${ }^{26,27}$, hematopoietic cell fate ${ }^{28,29}$, and carcinogenesis ${ }^{30}$. To our knowledge, there were several studies concerning the function of HOXA5 protein in cervical carcinoma. An analysis based on the TCGA database demonstrated that the up-regulation of HOXA5 is 
A

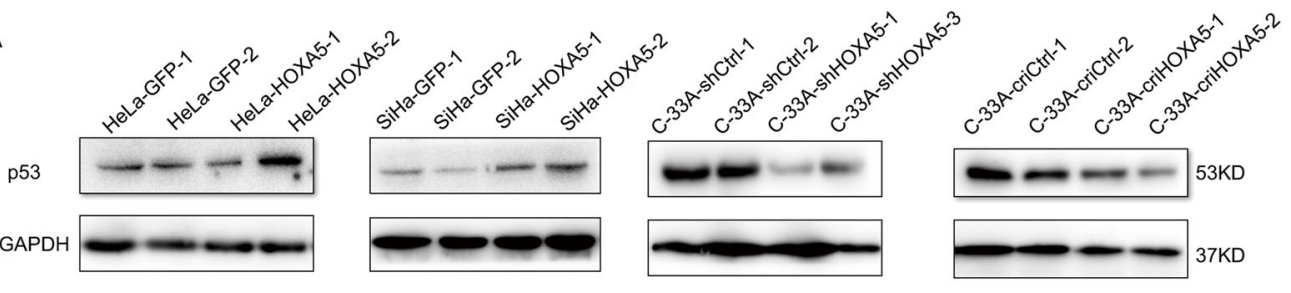

B

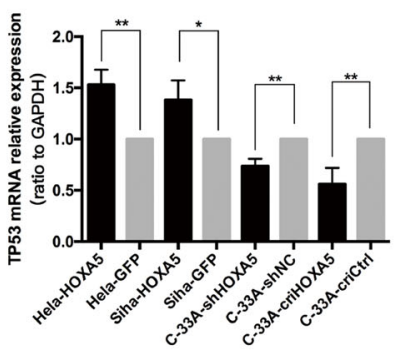

C

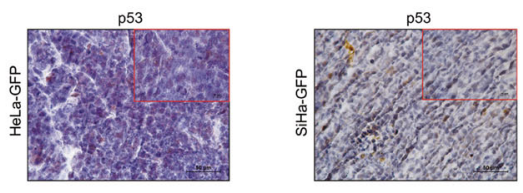

D

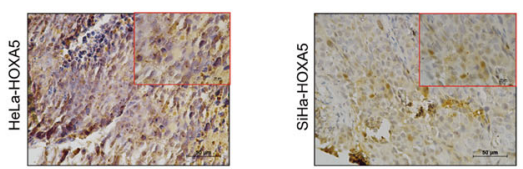

E
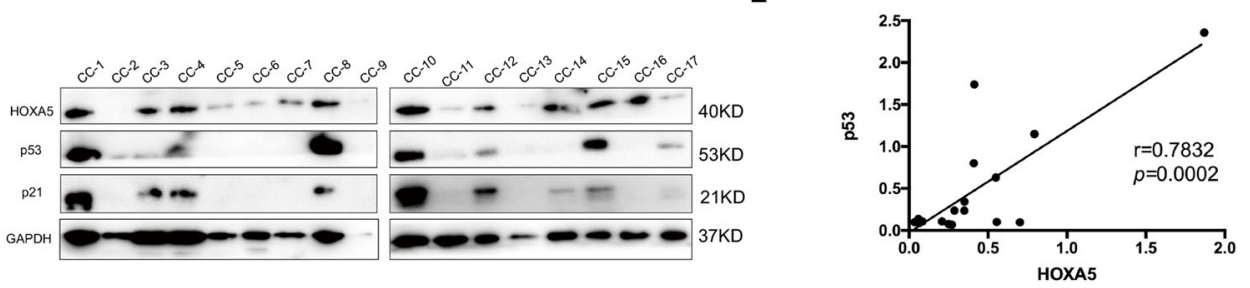

$\mathbf{F}$

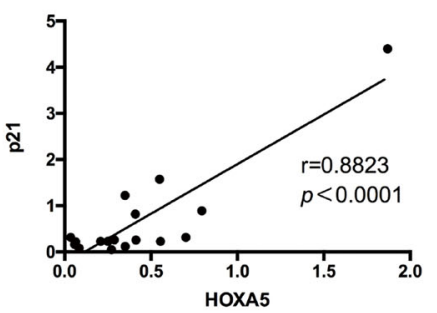

H

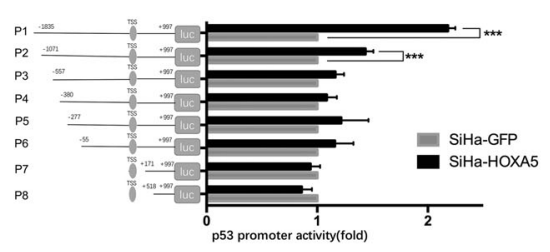

J
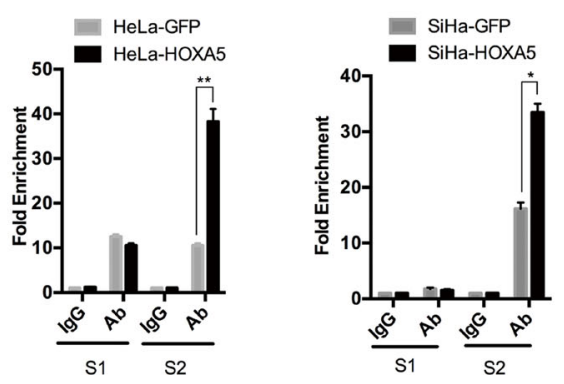

G

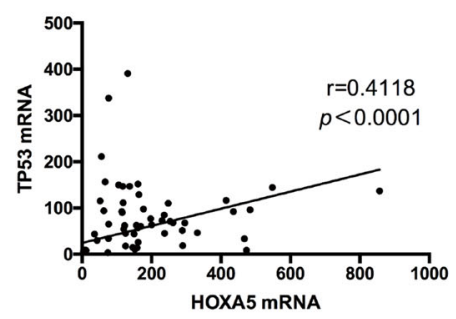

I

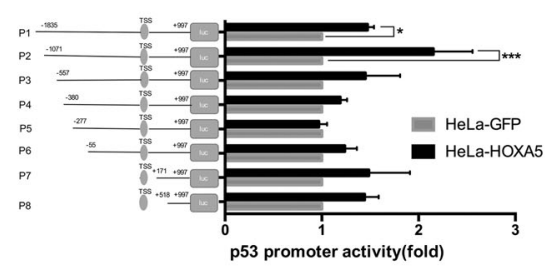

K

HOXA5 DNA-binding motif

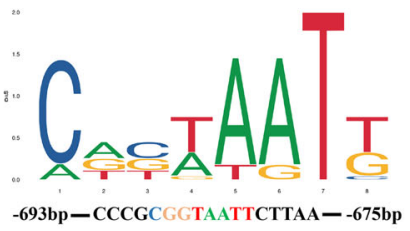

Fig. 7 (See legend on next page.) 
(see figure on previous page)

Fig. 7 HOXA5 regulates the expression of TP53 in cervical cancer cells. The expression of TP53 in HOXA5-modified cervical cancer cells was determined by real-time PCR (a) and western blot (b). The expression of p53 in xenograft was determined by IHC (c). d-f $\mathbf{f}$ The expression of HOXA5, p53, and p21 in clinical cervical cancer specimens was determined by western blot and the correlation was analyzed. $\mathbf{g}$ HOXA5 expression was significantly positively correlated with the expression of p53 $(r=0.4118, p<0.001)$ in cervical cancers (data from GEO database). $\mathbf{h}, \mathbf{i}$ The TP53 promoter structure was constructed and luciferase activity relative to Renilla control was measured in HeLa-HOXA5 and SiHa-HOXA5 cells. $\mathbf{j}$ The qChIP assay is shown in HeLa-HOXA5 and SiHa-HOXA5 cells immunoprecipitation by HOXA5 antibody and IgG antibody (as the negative control). k An experimentally defined transcription factor binding sites of HOXA5 was found in the JASPAR CORE database. The data were shown as the mean \pm SD of three independent experiments. ${ }^{*} p<0.05,{ }^{* *} p<0.01,{ }^{* * *} p<0.001$.

correlated with poor survival outcomes in cervical can$\mathrm{cer}^{31}$. However, Pei and colleagues found that HOXA5 was lowly expressed in cervical squamous cell carcinoma patients with poor differentiation ${ }^{32}$. Another report demonstrated that HOXA5 could suppress the proliferation and invasion and induce apoptosis through AKT and p27 in cervical cancer cells ${ }^{33}$.

Although the incidence of cervical cancer is decreasing globally, it is still the deadliest cancer in Africa and South America $^{1}$. It has been postulated that there is a consecutive progression from LSIL to HSIL and then to cervical carcinoma. In the present research, both the data from clinical specimens and the data from the GEO database indicated that the expression of HOXA5 protein was downregulated in cervical cancer. These results indicate HOXA5 is closely related with the neoplasia and development of cervical carcinoma.

Next, cell growth curves, MTT assays, and tumor xenografts showed that HOXA5 protein could inhibit tumor formation and progression by inhibiting cell proliferation. Furthermore, the cell cycle analysis revealed that HOXA5 could induce cell cycle arrest from G0/G1 phase to $S$ phase. These results demonstrate that HOXA5 inhibited cell proliferation by inducing cell cycle arrest from $\mathrm{G0} / \mathrm{G} 1$ phase to $\mathrm{S}$ phase.

The cell cycle is monitored by a set of cell cycle-related molecules. In different phases, diverse cell cycle proteins show different expression and degradation patterns, helping to coordinate the timing of every mitotic event ${ }^{34}$. In this study, functional experiments suggested that HOXA5 inhibited cell proliferation via inducing the cell cycle arrest from G0/G1 phase to $S$ phase. Our results showed that the expression of HOXA5 could induce obvious changes in cell cycle-related molecules, especially downregulation of cyclinD1 and upregulation of $\mathrm{p} 21$.

It has been reported that $\mathrm{Wnt} / \beta$-catenin signaling is related with the tumorigenesis in cervical carcinoma. Several studies have indicated $\mathrm{Wnt} / \beta$-catenin is activated in the development of cervical cancer ${ }^{35}$. Positive $\beta$-catenin staining was observed in $67.9 \%$ of cervical squamous cell carcinoma patients and the expression of it has a positive correlation with poor disease-free survival, as well as poor overall survival ${ }^{36}$. However, the underlying mechanism was not further elucidated. $\beta$-Catenin regulates a series of downstream target genes, including c-Myc and cyclinD1, which regulates the cell cycle process ${ }^{37}$. In the present study, the significant decrease in cyclinD1 prompted us to determine whether HOXA5 inhibited the expression of cyclinD1 via the Wnt/ $\beta$-catenin pathway. Our findings strongly demonstrate that HOXA5 inhibited the expression of cyclinD1 via the $\mathrm{Wnt} / \beta$-catenin pathway.

p53 is a crucial protein in cervical cancer progression. The HPV E6 oncoprotein can activate several mechanisms to downregulate p53 and interfere with p53 to block the function of the p53 protein ${ }^{38,39}$. Since p53 protein participates in complex biological processes in vivo, it can both induce the anti-proliferation and apoptosis effect in multiple tumors ${ }^{40}$. In the present study, another important cell cycle molecule that showed a significant change was the p21 protein. As reported previously, p21 is a welldefined downstream factor of the p53 pathway ${ }^{23}$. Therefore, we found both the data obtained from our clinical specimens and the data from the GEO database showed a strong correlation between HOXA5 and p53.

To further explore whether there is a direct relationship between HOXA5 and p53, a dual-luciferase assay was conducted. The results suggested HOXA5 could bind to the TAAT motif within the promoter of TP53 by its HD domain directly. The qChIP assay further confirmed that HOXA 5 binds between -693 and -675 in the promoter of TP53 and acts as a transcriptional activator to induce the expression of TP53. This is the first study demonstrating that HOXA5 affects the proliferation of cancer cells through both the Wnt/ $\beta$-catenin/cyclinD1 and p53/ p21 signaling pathways simultaneously.

In conclusion, our findings demonstrated that HOXA5 suppressed the activity of the Wnt/ $\beta$-catenin pathway and transactivated TP53 simultaneously, thus inhibiting the cell cycle progression by downregulating cyclinD1 and upregulating p21, ultimately inhibiting the neoplasia and progression in cervical cancer. 
A
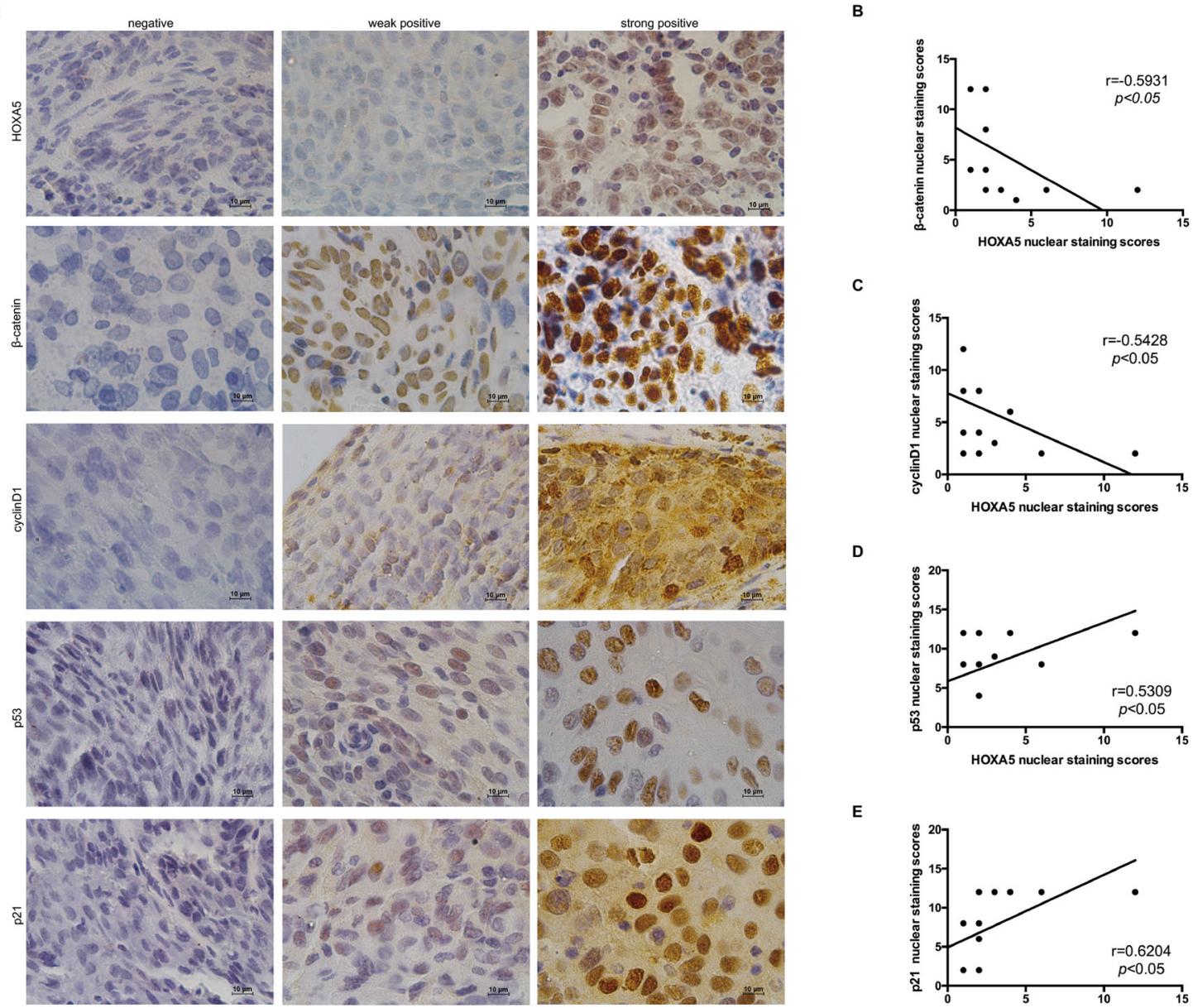

D
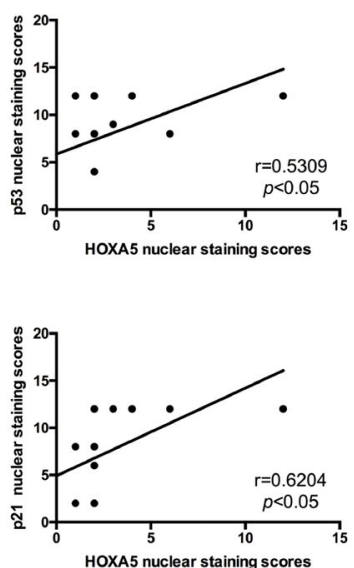

$\mathrm{F}$
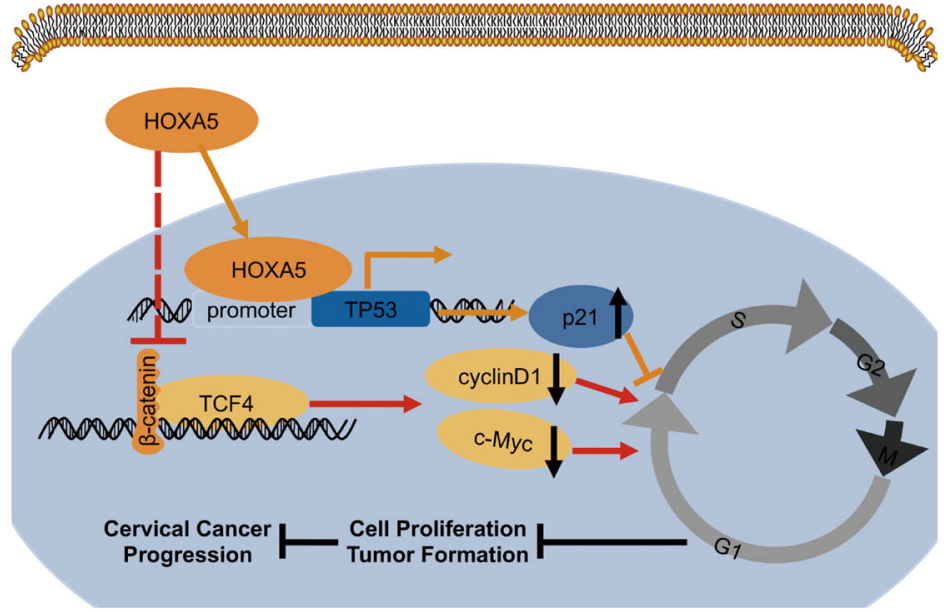

Fig. 8 Correlation analysis between the expression of HOXA5 with Wnt signaling and p53 in human cervical cancer specimens. a Fifteen cervical cancer specimens were analyzed by $\mathrm{HCC}$, and the representative images of HOXA5, $\beta$-catenin, cyclinD1, p53, and p21 were shown. b-e Correlation of the HOXA5 staining with $\beta$-catenin $(r=0.5931, p<0.05)$, c-Myc $(r=0.5186, p<0.05)$, cyclinD1 $(r=0.5428, p<0.05)$, TP53 $(r=0.5309$, $p<0.05)$, P21 $(r=0.6204, p<0.05)$. $\mathbf{f}$ The proposed mechanism of HOXA5 in cervical cancer cells. 


\section{Acknowledgements}

This work was supported by a grant to Prof. Peng-Sheng Zheng from the National Natural Science Foundation of China (Nos. 81472728 and 81672910).

\section{Conflict of interest}

The authors declare that they have no conflict of interest.

\section{Publisher's note}

Springer Nature remains neutral with regard to jurisdictional claims in published maps and institutional affiliations.

Supplementary Information accompanies this paper at (https://doi.org/ 10.1038/s41419-020-2629-3).

Received: 1 February 2020 Revised: 19 May 2020 Accepted: 21 May 2020 Published online: 04 June 2020

\section{References}

1. Bray, F. et al. Global cancer statistics 2018: GLOBOCAN estimates of incidence and mortality worldwide for 36 cancers in 185 countries. CA Cancer J. Clin. 68 , 394-424 (2018).

2. zur Hausen, H. Papillomaviruses and cancer: from basic studies to clinical application. Nat. Rev. Cancer 2, 342-350 (2002).

3. Mirabello, L. et al. HPV16 E7 genetic conservation is critical to carcinogenesis. Cell 170, 1164-1174 (2017).

4. Bhat, S., Kabekkodu, S. P., Noronha, A. \& Satyamoorthy, K. Biological implications and therapeutic significance of DNA methylation regulated genes in cervical cancer. Biochimie 121, 298-311 (2016).

5. Moody, C. A. \& Laimins, L. A. Human papillomavirus oncoproteins: pathways to transformation. Nat. Rev. Cancer 10, 550-560 (2010).

6. The Cancer Genome Atlas Research Network Integrated genomic and molecular characterization of cervical cancer. Nature 543, 378-384 (2017).

7. Li, L., Yang, W. T., Zheng, P. S. \& Liu, X. F. SOX17 restrains proliferation and tumor formation by down-regulating activity of the Wnt/beta-catenin signaling pathway via trans-suppressing beta-catenin in cervical cancer. Cell Death Dis. 9, 741 (2018).

8. Cui, N., Yang, W. T. \& Zheng, P. S. Slug inhibits the proliferation and tumor formation of human cervical cancer cells by up-regulating the p21/p27 proteins and down-regulating the activity of the Wnt/beta-catenin signaling pathway via the trans-suppression Akt1/p-Akt1 expression. Oncotarget $\mathbf{7}$ 26152-26167 (2016)

9. Yang, W. T. \& Zheng, P. S. Kruppel-like factor 4 functions as a tumor suppressor in cervical carcinoma. Cancer 118, 3691-3702 (2012).

10. Li, S., Ma, Y. M., Zheng, P. S. \& Zhang, P. GDF15 promotes the proliferation of cervical cancer cells by phosphorylating AKT1 and Erk1/2 through the receptor ErbB2. J. Exp. Clin. Cancer Res. 37, 80 (2018).

11. Liu, X. F., Li, X. Y., Zheng, P. S. \& Yang, W. T. DAX1 promotes cervical cancer cell growth and tumorigenicity through activation of Wnt/beta-catenin pathway via GSK3beta. Cell Death Dis. 9, 339 (2018).

12. Chen, Q., Zheng, P. S. \& Yang, W. T. EZH2-mediated repression of GSK-3beta and TP53 promotes Wnt/beta-catenin signaling-dependent cell expansion in cervical carcinoma. Oncotarget 7, 36115-36129 (2016).

13. Landry-Truchon, $\mathrm{K}$. et al. HOXA5 plays tissue-specific roles in the developing respiratory system. Development 144, 3547-3561 (2017).

14. Philippidou, P., Walsh, C. M., Aubin, J., Jeannotte, L. \& Dasen, J. S. Sustained Hox 5 gene activity is required for respiratory motor neuron development. Nat. Neurosci. 15, 1636-1644 (2012).

15. Landry-Truchon, K et al. Respiratory consequences of targeted losses of Hoxa5 gene function in mice. J. Exp. Biol. 220, 4571-4577 (2017).
16. Ordonez-Moran, P., Dafflon, C., Imajo, M., Nishida, E. \& Huelsken, J. HOXA5 counteracts stem cell traits by inhibiting Wnt signaling in colorectal cancer. Cancer Cell 28, 815-829 (2015).

17. Zhang, H., Zhao, J. H. \& Suo, Z. M. Knockdown of HOXA5 inhibits the tumorigenesis in esophageal squamous cell cancer. Biomed. Pharmacother. 86, 149-154 (2017)

18. Peng, X., Zha, L., Chen, A. \& Wang, Z. HOXA5 is a tumor suppressor gene that is decreased in gastric cancer. Oncol. Rep. 40, 1317-1329 (2018).

19. Yoo, K. H., Park, Y. K., Kim, H. S., Jung, W. W. \& Chang, S. G. Epigenetic inactivation of HOXA5 and MSH2 gene in clear cell renal cell carcinoma. Pathol. Int. 60, 661-666 (2010).

20. Wang, Y. F. et al. Cooperativity of HOXA5 and STAT3 is critical for HDAC8 inhibition-mediated transcriptional activation of PD-L1 in human melanoma cells. J. Investig. Dermatol. 138, 922-932 (2018).

21. Teo, W. W. et al. HOXA5 determines cell fate transition and impedes tumor initiation and progression in breast cancer through regulation of E-cadherin and CD24. Oncogene 35, 5539-5551 (2016).

22. Zeng, Y. T., Liu, X. F., Yang, W. T. \& Zheng, P. S. REX1 promotes EMT-induced cell metastasis by activating the JAK2/STAT3-signaling pathway by targeting SOCS1 in cervical cancer. Oncogene 38, 6940-6957 (2019).

23. Kastenhuber, E. R. \& Lowe, S. W. Putting p53 in context. Cell 170, 1062-1078 (2017).

24. Bulatov, E. et al. Isatin-Schiff base-copper (II) complex induces cell death in p53-positive tumors. Cell Death Discov. 4, 103 (2018).

25. Joshi, R. et al. Functional specificity of a Hox protein mediated by the recognition of minor groove structure. Cell 131, 530-543 (2007).

26. Aubin, J., Lemieux, M., Tremblay, M., Behringer, R. R. \& Jeannotte, L. Transcriptional interferences at the Hoxa4/Hoxa5 locus: importance of correct Hoxa5 expression for the proper specification of the axial skeleton. Dev. Dyn. 212, 141-156 (1998).

27. Joksimovic, M., Jeannotte, L. \& Tuggle, C. K. Dynamic expression of murine HOXA5 protein in the central nervous system. Gene Expr. Patterns 5, 792-800 (2005).

28. Crooks, G. M. et al. Constitutive HOXA5 expression inhibits erythropoiesis and increases myelopoiesis from human hematopoietic progenitors. Blood $\mathbf{9 4}$, 519-528 (1999).

29. Sugimura, R. et al. Haematopoietic stem and progenitor cells from human pluripotent stem cells. Nature 545, 432-438 (2017).

30. Chen, H. et al. HOXA5 acts directly downstream of retinoic acid receptor beta and contributes to retinoic acid-induced apoptosis and growth inhibition. Cancer Res. 67, 8007-8013 (2007).

31. Eoh, K. J. et al. Upregulation of homeobox gene is correlated with poor survival outcomes in cervical cancer. Oncotarget 8, 84396-84402 (2017).

32. Pei, L. et al. Expression and clinical significance of HOXA5, E-cadherin, and beta-catenin in cervical squamous cell carcinoma. Int. J. Clin. Exp. Pathol. 11, 3091-3096 (2018).

33. Wang, Z., Yu, C. \& Wang, H. HOXA5 inhibits the proliferation and induces the apoptosis of cenvical cancer cells via regulation of protein kinase B and p27. Oncol. Rep. 41, 1122-1130 (2019).

34. Schafer, K. A. The cell cycle: a review. Vet. Pathol. 35, 461-478 (1998).

35. Bahrami, A. et al. Clinical significance and prognosis value of Wnt signaling pathway in cervical cancer. J. Cell. Biochem. 118, 3028-3033 (2017).

36. Liang, J. et al. beta-Catenin expression negatively correlates with WIF1 and predicts poor clinical outcomes in patients with cervical cancer. Biomed. Res. Int. 2016, 4923903 (2016).

37. Clevers, H. \& Nusse, R. Wnt/beta-catenin signaling and disease. Cell 149, 1192-1205 (2012)

38. Scheffner, M., Werness, B. A., Huibregtse, J. M., Levine, A. J. \& Howley, P. M. The E6 oncoprotein encoded by human papillomavirus types 16 and 18 promotes the degradation of p53. Cell 63, 1129-1136 (1990).

39. Scheffner, M., Huibregtse, J. M., Vierstra, R. D. \& Howley, P. M. The HPV-16 E6 and E6-AP complex functions as a ubiquitin-protein ligase in the ubiquitination of p53. Cell 75, 495-505 (1993).

40. Inoue, K., Fry, E. A. \& Frazier, D. P. Transcription factors that interact with p53 and Mdm2. Int. J. Cancer 138, 1577-1585 (2016). 